\title{
Study of a Closed Divertor Configuration for the Three-Dimensionally Complicated Magnetic Structure in the LHD Plasma Periphery
}

\author{
Mamoru SHOJI, Masahiro KOBAYASHI, Suguru MASUZAKI, Atsushi IWAMAE ${ }^{1)}$, \\ Tsuguhiro WATANABE, Hiroshi YAMADA, Akio KOMORI and LHD experimental groups \\ National Institute for Fusion Science, 322-6 Oroshi-cho, Toki 509-5292, Japan \\ 1)Department of Engineering Physics and Mechanics, Graduate School of Engineering, Kyoto University, \\ Yoshida-Honmachi, Sakyo-ku, Kyoto 606-8501, Japan
}

(Received 15 November 2007 / Accepted 19 March 2008)

\begin{abstract}
The distribution of strike points in a closed divertor configuration with additional baffle plates installed in the toroidal ends of closed divertor components near lower/upper ports is calculated by tracing magnetic field lines from the last closed flux surface in various magnetic configurations (radial position of the magnetic axis, $R_{\mathrm{ax}}=3.50 \sim 3.90 \mathrm{~m}$ ). The calculation shows that the ratio of the number of strike points on additional plates is maximum for $R_{\mathrm{ax}}=3.60 \mathrm{~m}$, showing that the plates are effective in this magnetic configuration. Neutral particle transport is investigated using a fully three-dimensional code EIRENE with one-dimensional plasma fluid analysis of divertor legs, where the plasma parameter profiles on the legs are obtained by an iterative calculation including interaction processes between the plasma and neutral particles. The plates raise the pressure of molecular hydrogen locally near the baffle plates to more than $0.2 \mathrm{~Pa}$, which is enough for efficient particle control using vacuum pumping systems installed near the plates. The simulation proposes one possible candidate of optimized closed divertor configuration for the three-dimensionally complicated magnetic structure in the LHD plasma periphery.
\end{abstract}

(C) 2008 The Japan Society of Plasma Science and Nuclear Fusion Research

Keywords: plasma edge transport, neutral particle transport, EIRENE, closed divertor, plasma fluid analysis, strike point, baffle plate

DOI: $10.1585 /$ pfr.3.S1038

\section{Introduction}

Control of the plasma density and neutral particles in the LHD plasma periphery is experimentally found to be essential for achieving super dense core (SDC) plasmas and sustaining long pulse discharges [1]. In the phase II experimental campaign, a closed divertor configuration with vacuum pumping systems is planned for efficient particle control. Magnetic components produced by two super conducting coils (helical and poloidal) form a threedimensionally complicated magnetic field line structure in the plasma periphery. Therefore, we started the study of the design of the closed divertor configuration optimized to the complicated magnetic structure using a fully threedimensional neutral particle transport code (EIRENE) with one-dimensional plasma fluid analysis of divertor legs [2].

Previous neutral particle transport analysis in various magnetic configurations strongly suggests that installation of closed divertor components, which consist of baffle plates, a dome structure, and slanted divertor plates installed along the space between two helical coils in the inboard side of the torus, is reasonable for efficient particle control, because the density of neutral particles is relatively high in the inboard side. The simulation results are in good agreement with the measurements of polarization-resolved $\mathrm{H}_{\alpha}$ spectra and vertical profiles of the line integrated $\mathrm{H}_{\alpha}$ intensity [3]. The simulation predicts that the density of neutral hydrogen molecules in the inboard side for the closed divertor configuration increases by more than one order of magnitude compared to that in the present opened divertor case, which seems to be marginally acceptable for efficient particle control using vacuum pumping systems.

Installation of additional baffle plates at the toroidal ends of the closed divertor components near lower/upper ports can effectively contribute to further enhancement of the neutral particle density. This is because the additional baffle plates can confine neutral particles in the inboard side, and the plates change the position of strike points (particle sources) from the lower/upper side to the inboard side by intersecting divertor legs similar to a target plate.

This paper proposes one possible candidate for the optimized closed divertor configuration. The effect of the additional baffle plates on particle control in the LHD plasma periphery is investigated by magnetic field line tracing and using the three-dimensional neutral particle transport code. 


\section{Magnetic Field Line Configuration in the Plasma Periphery}

The magnetic configuration in the LHD plasma is three-dimensionally complicated. Helically twisted plasmas are formed by the magnetic field produced by superconducting helical and poloidal coils. An ergodic layer consisting of complicated magnetic field lines is formed around the last closed magnetic flux surface (LCFS). Four bundled magnetic field lines (divertor legs) are deviated from the ergodic layer at the position of X-points. Magnetic field lines on the divertor legs connect directly to divertor plates (carbon) or the vacuum vessel (stainless steel). The magnetic configurations in the plasma periphery are changed significantly with the radial position of the magnetic axis $R_{\mathrm{ax}}$, which is controlled by the electric current in the superconducting coils. For designing an optimized closed divertor configuration, we have to consider the three-dimensionally complicated structure of the magnetic field lines.

The spatial profile and density of neutral particles are essential parameters for optimizing the divertor configuration. Toroidal and poloidal distributions of the particle source are key parameters for determining the spatial profile of the neutral particle density. The measurement of the $\mathrm{H}_{\alpha}$ emission profile is a standard technique for estimating the neutral density profile and distribution of the particle source. Figure 1 (a) shows the images of $\mathrm{H}_{\alpha}$ emission near a lower port (2.5-L), which is observed with a tan- (a)
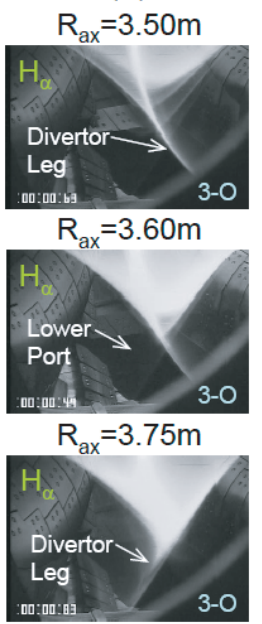

$R_{a x}=3.90 m$

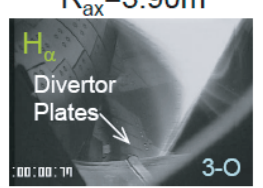

(b)

(c)

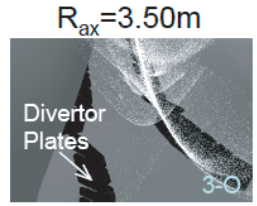

$\mathrm{R}_{\mathrm{ax}}=3.60 \mathrm{~m}$

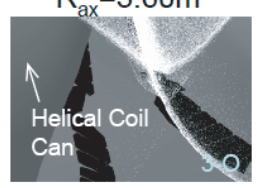

$\mathrm{R}_{\mathrm{ax}}=3.75 \mathrm{~m}$

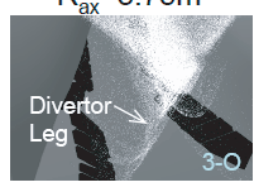

$\mathrm{R}_{\mathrm{ax}}=3.90 \mathrm{~m}$
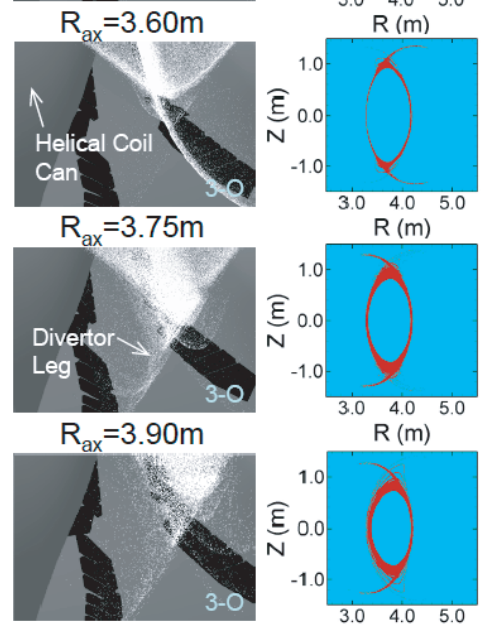
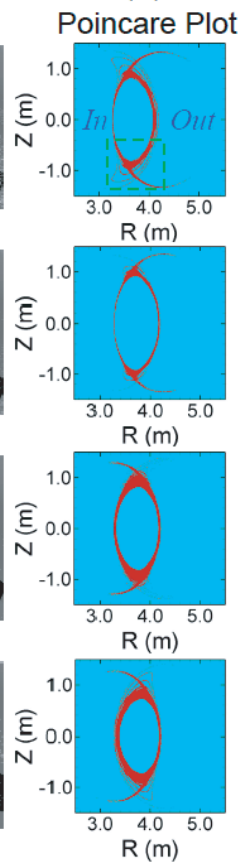

Fig. 1 Images of $\mathrm{H}_{\alpha}$ emission near a lower port (a) and threedimensional plots of the magnetic field lines in the plasma periphery (white dots) (b), and Poincare plots at a toroidal angle where the shape of the plasma is vertically elongated (c) in various magnetic configurations $\left(\mathrm{R}_{\mathrm{ax}}=3.50 \mathrm{~m} \sim 3.90 \mathrm{~m}\right)$.

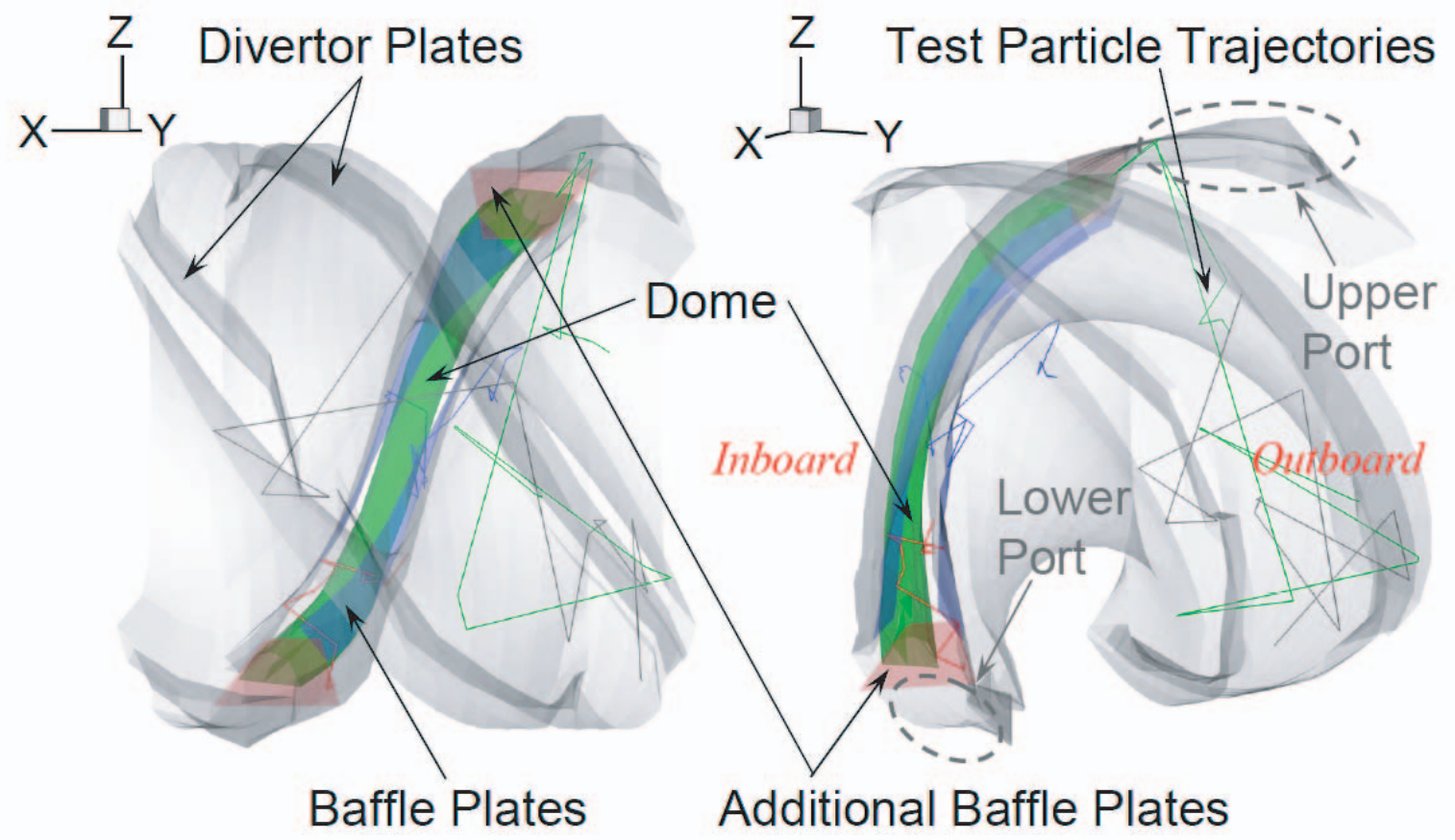

Fig. 2 Three-dimensional model for neutral particle transport simulation in the closed divertor configuration with additional baffle plates (target plates) installed at both toroidal ends of a dome structure in the inboard side of the torus. The closed divertor consists of baffle plates, a dome structure, and slanted divertor plates, which are installed in the inboard side of the torus. Test particle trajectories calculated using neutral particle transport simulation are also shown. 
gentially viewing visible CCD camera installed in an outer port (3-O) in various magnetic configurations. The images indicate that the $\mathrm{H}_{\alpha}$ emission profile on the divertor legs is changed significantly with the magnetic configuration, which suggests that the distribution of the plasma flow onto the divertor plates changes with $R_{\mathrm{ax}}$. For interpreting the observed images from the viewpoint of the magnetic field line structure in the plasma periphery, the magnetic field lines are traced from positions on the LCFS in the threedimensional model including the geometry of the vacuum vessel, helical coils, and divertor plates. Figure 1 (b) shows the images of three-dimensional plots of the magnetic field lines near the lower port, which are viewed from the position of the camera. Figure 1 (c) shows Poincare plots on the poloidal plane at a toroidal angle where the shape of the LHD plasma is vertically elongated. A green broken square in the plot $\left(R_{\mathrm{ax}}=3.50 \mathrm{~m}\right)$ represents the observation area of the CCD camera. The magnetic field line configuration in the divertor legs on this poloidal plane changes significantly with $R_{\mathrm{ax}}$. Although most of the magnetic field lines in $R_{\mathrm{ax}}=3.50$ and $3.60 \mathrm{~m}$ are bundled to the outboard side of the torus, the magnetic field lines are almost connected to the inboard side of the torus in the other magnetic configurations $\left(R_{\mathrm{ax}}=3.75\right.$ and $\left.3.90 \mathrm{~m}\right)$ (see Fig. 1 (c)).

Images of the three-dimensional plots of the magnetic field lines are consistent with the $\mathrm{H}_{\alpha}$ measurements, which indicates that the distribution of the plasma flow onto the divertor plates can be estimated by the calculation of the distribution of strike points. It is experimentally supported by the toroidal and poloidal distributions of the temperature increments of the divertor plates during plasma discharges measured with thermocouples embedded in the divertor plates. The profile of the ion saturation current on a divertor plate measured with Langmuir probes also shows reasonable agreement with the calculation in various magnetic configurations [4].

\section{Calculations of the Distribution of Strike Points}

Neutral particle transport simulation has shown that the density of neutral hydrogen molecules in the inboard side of the torus on the poloidal plane where the shape of the plasma is horizontally elongated is higher than that in the other regions for $R_{\mathrm{ax}}=3.60 \mathrm{~m}$. In this simulation, the toroidal and poloidal distribution of the neutral particle source is regarded as that of the strike points calculated by the magnetic field line tracing. The reason for the formation of the high density of hydrogen molecules is that the strike points are concentrated in the inboard side of the torus on this poloidal plane for $R_{\mathrm{ax}}=3.60 \mathrm{~m}$, and the three-dimensional geometry of the vacuum vessel also contributes to formation of the high molecular density. This prediction is consistent with the measurements with a multi-channel vertical $\mathrm{H}_{\alpha}$ detector array, which consists of a high wavelength resolution spectrometer and po-

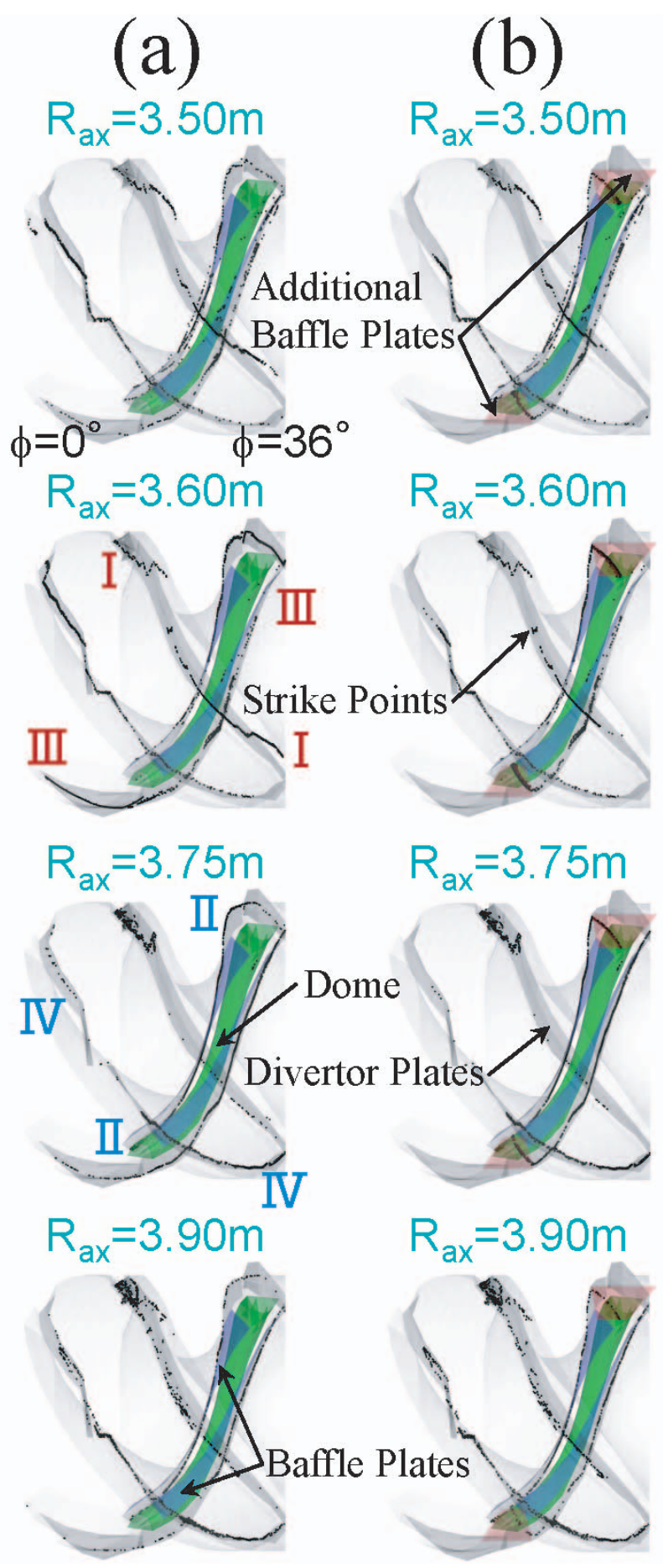

Fig. 3 Toroidal and poloidal distribution of strike points (black dots) in the closed divertor configuration without (a) and with (b) additional baffle plates in various magnetic configurations $\left(\mathrm{R}_{\mathrm{ax}}=3.50 \mathrm{~m} \sim 3.90 \mathrm{~m}\right)$.

larization separation optics [5]. The position and intensity of $\mathrm{H}_{\alpha}$ emission, and the flow velocity and temperature of neutral particles locating on the line of sight of the detectors are estimated by fitting the polarization-resolved $\mathrm{H}_{\alpha}$ spectra (e- and o-ray) using least-squares fitting with four sets of Zeeman profiles and a broad Gaussian profile. The analysis has shown that the $\mathrm{H}_{\alpha}$ emission in the inboard 
side of the torus at a poloidal plane where the shape of the LHD plasma is horizontally elongated is significantly higher than that in the outboard side for $R_{\mathrm{ax}}=3.60 \mathrm{~m}$. The calculations of the polarization-resolved $\mathrm{H}_{\alpha}$ spectra, which are obtained by the density profile and velocity vector of neutral particles, configuration of the magnetic field lines, and geometrical arrangement between the detectors and the emission area, agree well with the measurements. The neutral particle transport simulation also predicts the formation of a high density of neutral hydrogen molecules in the outboard side for $R_{\mathrm{ax}}=3.90 \mathrm{~m}$. The calculations also agree with the measurements using the $\mathrm{H}_{\alpha}$ detector array in this magnetic configuration. The vertical profiles of the line integrated $\mathrm{H}_{\alpha}$ intensity along the line of sight of the detectors agree well with the calculated profiles in various magnetic configurations. The consistency of the polarization-resolved $\mathrm{H}_{\alpha}$ spectra and line integrated $\mathrm{H}_{\alpha}$ intensity profiles with the measurements prove that the simulation is effectively applicable to a detailed analysis of neutral particle transport in the LHD plasma periphery and the study of the optimized closed divertor configuration.

Good plasma confinement with excellent plasma parameters has been achieved successfully in a magnetic configuration of $R_{\mathrm{ax}}=3.60 \mathrm{~m}$. As the neutral density in this magnetic configuration is high in the inboard side of the torus, installation of closed divertor components in the inboard side is reasonable for efficient particle control. The optimization of the closed divertor configuration was per- formed by trial and error, which found that baffle plates located near the divertor plates enhance the neutral density in the inboard side. A dome structure between the two helical coils and slanted divertor plates also contributes to the increase in the neutral density. These three components effectively lead neutral particles to the backside of the dome structure by particle reflection due to the baffle plates [2]. Figure 2 shows two figures of a three-dimensional model of the closed divertor components with the additional baffle plates (carbon) for the neutral particle transport simulation. The left figure is the model viewed from an outer port, which shows all the closed divertor components installed in the inboard side of the torus. The right figure is a bird's eye view of the components for displaying the shape and configuration of an additional baffle plate installed near a lower port. Test particle trajectories (representatives of a neutral particle trajectory) calculated using the neutral particle transport code are also shown in these figures.

Figure 3 (a) illustrates the calculations of the distribution of strike points (black dots) in various magnetic configurations $\left(R_{\mathrm{ax}}=3.50 \mathrm{~m} \sim 3.90 \mathrm{~m}\right)$ for one toroidal pitch angle $\left(0^{\circ} \sim 36^{\circ}\right)$. Figure $3(\mathrm{~b})$ shows the distribution of strike points in the case with the additional baffle plates installed at both the toroidal ends of the closed divertor components. The additional baffle plate functions like a target plate that intersects a divertor leg in order to change the distribution of strike points originating from one of the divertor legs, which contributes to concentration of strike
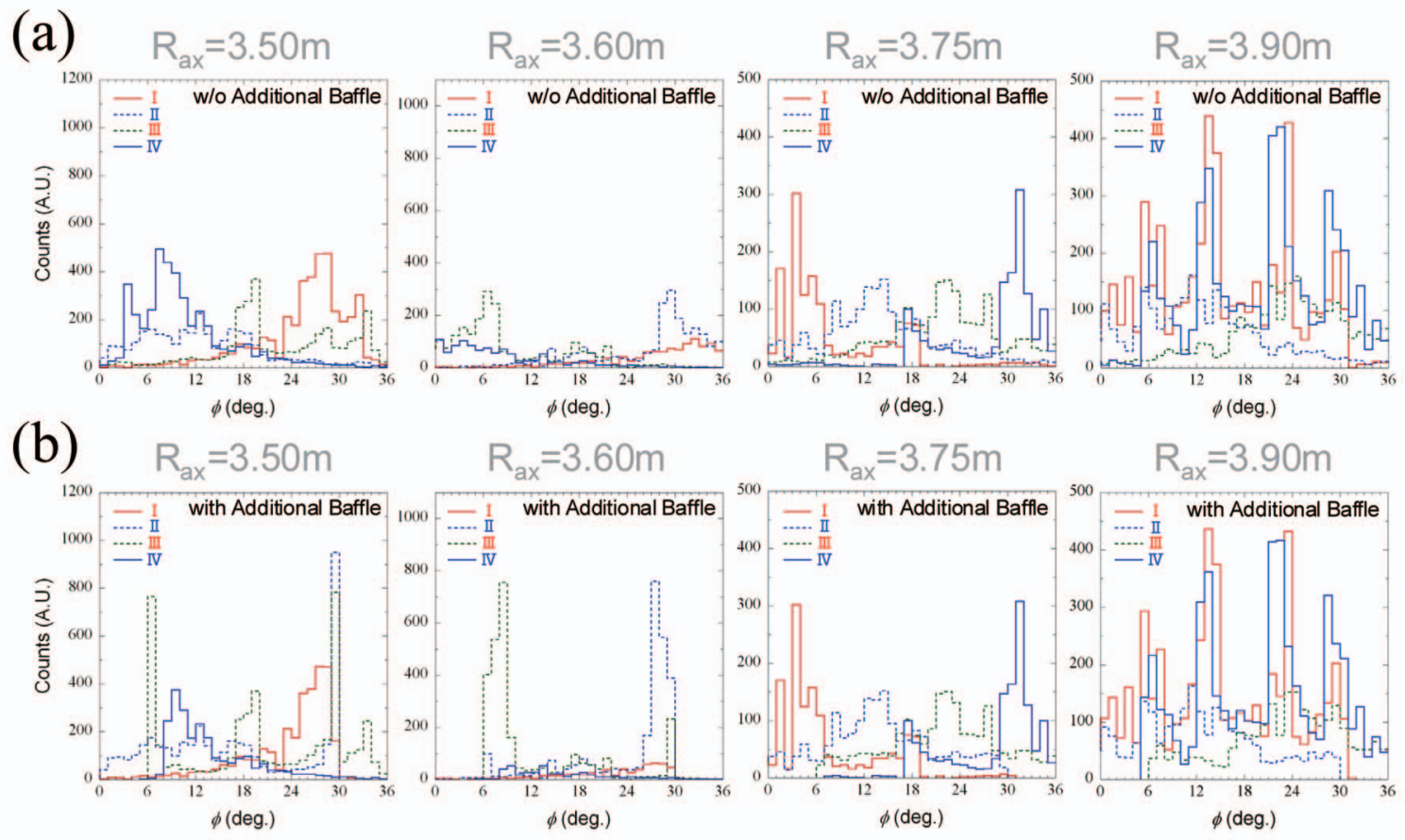

Fig. 4 Histograms of the number of strike points along four divertor legs in the case with (a) and without the additional baffle plates (b) in various magnetic configurations $\left(\mathrm{R}_{\mathrm{ax}}=3.50 \sim 3.90 \mathrm{~m}\right)$. The toroidal and poloidal positions of the strike points along the four divertor legs (I, II, III, IV) are indicated in Fig. 3 (a). 
points in the inboard side of the torus. The shape of the additional baffle plates is designed so that they do not interfere with other divertor legs. This is because the magnetic field lines on other divertor legs connect to the inboard side of the torus, which is one of the main particle sources for enhancing the neutral density in the inboard side.

Figures 4 (a) and (b) represent the histograms of the number of strike points along four divertor legs in the case with and without the additional baffle plates, respectively. The baffle plates vary the toroidal distribution of strike points in the ranges of the toroidal angle $\left(0^{\circ}<\phi<8^{\circ}\right.$ and $28^{\circ}<\phi<36^{\circ}$ ). The distribution of the number of strike points along the divertor legs II and III (shown in Fig. 3) in the magnetic configurations $\left(R_{\mathrm{ax}}=3.50\right.$ and $\left.3.60 \mathrm{~m}\right)$ is significantly influenced by the additional baffle plates. The strike points are concentrated in the toroidal angles where the additional plates are installed. The baffle plates are not effective for changing the distribution of strike points in the magnetic configurations $\left(R_{\mathrm{ax}}=3.75\right.$ and $\left.3.90 \mathrm{~m}\right)$. Slight change of the number of strike points along the divertor legs (II and III) appears in the ranges of the toroidal angle. This is because the number of the magnetic field lines that connect directly to the additional baffle plates is quite low in these magnetic configurations.

Figure 5 shows the ratio of the number of strike points on the additional baffle plates to that of all strike points. It indicates that the ratio is maximum (more than 0.6) for $R_{\mathrm{ax}}=3.60 \mathrm{~m}$, which means that the main neutral particle sources are located on the additional baffle plates. It shows that the plates are quite effective in this magnetic configuration, which is typical for achieving high plasma parameters with good plasma confinement. The reason for the increase in the ratio with $R_{\mathrm{ax}}$ for $R_{\mathrm{ax}} \leq 3.60 \mathrm{~m}$ is the change in the structure of peripheral magnetic field lines

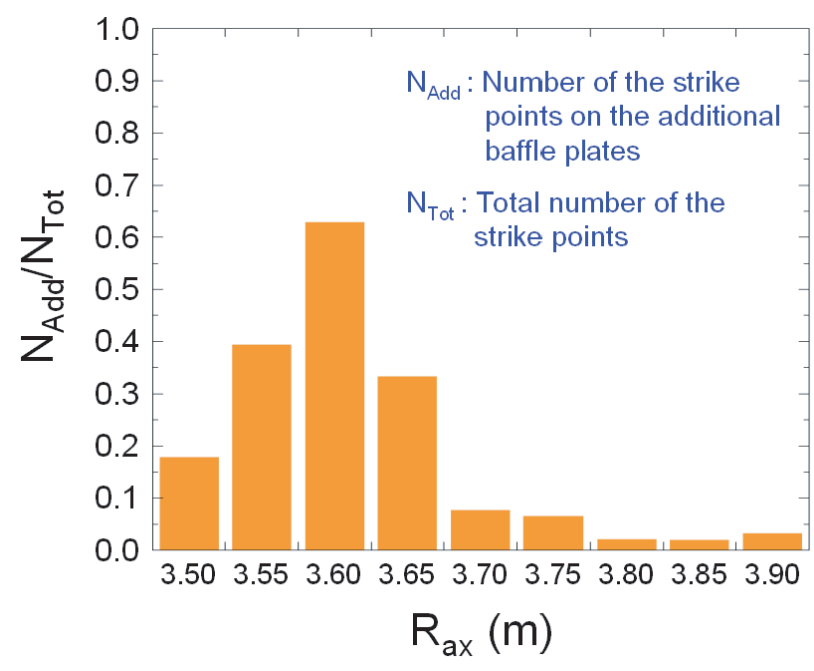

Fig. 5 Ratio of the number of strike points on the additional baffle plates to that of all strike points in various magnetic configurations $\left(\mathrm{R}_{\mathrm{ax}}=3.50 \sim 3.90 \mathrm{~m}\right)$. in the inboard side of the torus, which induces the concentration of strike points on the additional baffle plates. The magnetic field line structure changes significantly with $R_{\mathrm{ax}}$ in the case where $R_{\mathrm{ax}}>3.65 \mathrm{~m}$. Most of the magnetic field lines in the inboard side are bundled into the divertor legs that are not connected directly to the additional baffle plates. This is the main reason for the rapid decrease in the ratio with $R_{\mathrm{ax}}$.

\section{Neutral Particle Transport Sim- ulation with a One-dimensional Plasma Fluid Analysis}

EMC3-EIRENE code has been used widely for detailed analysis of the plasma and neutral particle transport in the peripheral plasma in helical/stellarator and tokamak devices [6]. This sophisticated analysis using a fully three-dimensional plasma fluid code coupled with the neutral particle transport code is too time consuming in our computational environment. Furthermore, the code does not include the magnetic field line structures on divertor legs due to some technical reasons. In order to overcome the above two restrictions, we extend the calculation domain so as to include the divertor legs with an assumption that the plasma parameter profiles inside the ergodic layer are fixed during the iteration process between the plasma and neutral particle transport. Following three differential equations for plasma density, momentum, and energy are solved along the magnetic field lines on the divertor legs by the Runge-Kutta method from the upstream of the divertor legs (in the proximity of $\mathrm{X}$-points in the ergodic layer) to divertor plates [7],

$$
\begin{aligned}
& \frac{\mathrm{d}\left(n v_{/ /}\right)}{\mathrm{d} s}=S_{\mathrm{p}}, \\
& \frac{\mathrm{d}}{\mathrm{d} s}\left(2 n T+m n v_{/ /}^{2}\right)=-m v_{/ /}<\sigma v>_{\mathrm{cx}} n n_{0}, \\
& \frac{\mathrm{d}}{\mathrm{d} s}\left(5 n T v_{/ /}-\kappa_{/ /}^{0} T^{2.5} \frac{\mathrm{d} T}{\mathrm{~d} s}\right)=Q_{\text {loss }},
\end{aligned}
$$

where $S_{\mathrm{p}},\langle\sigma v\rangle_{\mathrm{cx}}, n_{0}, Q_{\text {loss }}$ are the particle source, rate coefficient of charge exchange, neutral density, and energy loss via ionization obtained using the neutral particle transport code, respectively. The parameter $\kappa_{/ /}^{0} T^{2.5}$ indicates plasma heat conductivity along a magnetic field line on the divertor legs. In the above three differential equations, electron temperature is assumed to be the same as ion temperature, and the effects of impurities are neglected.

The connection length of the magnetic field lines on divertor legs is very short (less than several meters), where one-dimensional plasma fluid analysis is applicable, because the plasma transport transverse to the magnetic field lines is negligible [8]. Iterative calculation of the plasma parameter profiles on the divertor legs coupled with the parameter profiles of neutral particles (hydrogen atoms and molecules) gives a converged solution under the following two boundary conditions within a reasonable computation 
time:

1. The plasma flow velocity $\left(v_{/}\right)$at the surface of the divertor plates is fixed to the sound speed $c_{\mathrm{s}}$ for satisfying the Bohm criterion,

2. Three invariants (plasma density, momentum, and energy) at the upstream of the divertor legs are fixed to be the values in the ergodic layer near the X-points, which are calculated using the EMC3-EIRENE code in the case of $P_{\text {input }}=8 \mathrm{MW}, n_{\mathrm{e}}^{\mathrm{LCFS}} \sim 4 \times 10^{19} \mathrm{~cm}^{-3}$, $\Gamma_{\text {total }}=3.6 \times 10^{4} \mathrm{~A}$.

Conversion of the one-dimensional plasma parameter profiles along the magnetic field lines on the divertor legs to that in the three-dimensional grid model in the EIRENE code is based on the procedure used in a track-length estimator [9]. The plasma parameters in a grid are calculated from the total path length of magnetic field lines in the grid, parameter profile along the magnetic field line, and grid volume.

\section{Calculations of the Density Profile of Neutral Particles in the Opti- mized Closed Divertor Configura- tion}

Figure 6 (a) shows four poloidal cross sections of the calculated density profile of neutral hydrogen molecules for the closed divertor configuration (without the additional baffle plates) for $R_{\mathrm{ax}}=3.60 \mathrm{~m}$, showing enhancement of neutral density in the inboard side of the torus at the back side of the dome structure $\left(\phi=18^{\circ}\right)$. In this calculation, the absolute neutral density is strongly dependent on the particle reflection ratio of the divertor plates.
We determined the ratio $\left(R_{\text {div }}\right)$ to be 0.3 so that the neutral pressure of the molecular hydrogen in the inboard side is of the order of $1 \mathrm{mPa}$ for the present opened divertor configuration. This neutral pressure agrees roughly with measurements with a fast ion gauge during typical plasma discharges in this magnetic configuration [10].

The simple particle balance analysis during fuel pellet injection indicates that increase in neutral pressure of hydrogen molecules up to about $0.1 \mathrm{~Pa}$ is favorable for efficient particle control. The neutral particle transport simulation indicates that the neutral pressure in the inboard side is expected to be about $20 \mathrm{mPa}\left(n_{\mathrm{H} 2} \sim 5 \times 10^{12} \mathrm{~cm}^{-3}\right)$ in the closed divertor configuration, which seems to be marginally acceptable for particle control in the plasma periphery. Further enhancement of the pressure of neutral particles is strongly desired for improving the efficiency of particle pumping.

The additional baffle plates are introduced into the three-dimensional grid model for the neutral particle transport simulation. We can set arbitrary shaped plates using the function of an "additional surface" in EIRENE code. The additional plates are defined by some points (more than two) of Cartesian coordinate in the grid model [11]. The released particles from the additional baffle plates are assumed to be neutral hydrogen molecules with the energy corresponding to the room temperature $(300 \mathrm{~K})$, and the direction of the released molecules is assumed to be normal to the plates. The number of released hydrogen molecules is determined from the particle reflection ratio, and the plasma flux is calculated by one-dimensional plasma fluid analysis of the divertor legs. The position of the released molecules from the additional baffle plates is determined by averaging the coordinates of some strike points on the

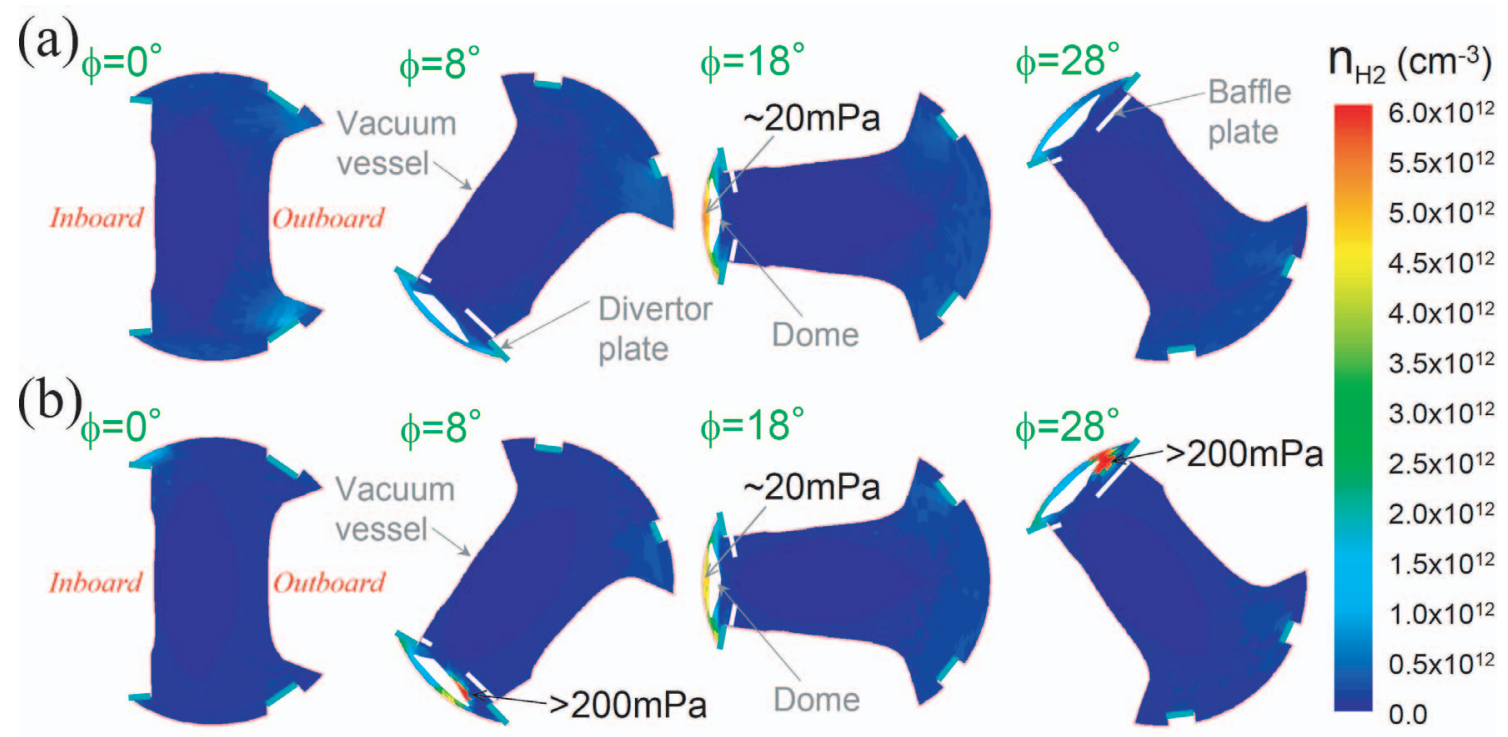

Fig. 6 Four poloidal cross-sections of the calculated density profile of neutral hydrogen molecules for the closed divertor configuration (a) and the configuration with the additional baffle plates $\left(\mathrm{R}_{\mathrm{ax}}=3.60 \mathrm{~m}\right)(\mathrm{b})$. 
additional baffle plates (point neutral sources are assumed). In the closed divertor components (except for the additional baffle plates), the quantity, profile, and species of the released neutral particles are based on the TRIM code.

Figure 6(b) shows four poloidal cross sections of the density profile of neutral hydrogen molecules for the closed divertor configuration with the additional baffle plates. It indicates that the pressure of neutral hydrogen molecules in the inboard side of the tours is about $20 \mathrm{mPa}$, which is not different from that in the original closed divertor configuration (shown in Fig.6(a)). The calculation shows that the molecular hydrogen density is locally high on the dome structure near both the additional baffle plates, as shown in the figure at the toroidal angles $\phi$ $=8^{\circ}$ and $28^{\circ}$. The mean free path of the neutral hydrogen molecules in the divertor legs near the additional baffle plates is estimated to about $4 \mathrm{~mm}$, which is quite shorter than the typical width of the divertor legs near the baffle plates $(\sim 20 \mathrm{~mm})$. Helically twisted shape of the vacuum vessel and divertor legs is effective to ionize the neutral hydrogen molecules released from the additional baffle plates because the molecules cannot travel in the twisted divertor region without interacting with the divertor plasma. Small conductance between the divertor plasma and dome structure can also prevent diffusion of molecules in the divertor region. Due to the configuration of the magnetic field lines on the divertor legs, most of the ionized molecules released from the additional baffle plates return to the plates. The small particle reflection ratio (0.3) reduces the recycling of hydrogen molecules in the divertor region. The local formation of high molecular density near the additional baffle plates can be explained by the above reasons. The neutral pressure becomes greater than $0.20 \mathrm{~Pa}$, which is sufficient for efficient particle control in the plasma periphery. The heat load onto the additional baffle plates is estimated to be about $4 \mathrm{MW} / \mathrm{m}^{2}$ in this calculation condition. This heat load seems to be manageable by improving existing divertor plate structure and heat removal system in LHD. Inclining the plates may contribute to the reduction of the heat load by extending the plasma wetted area.

The neutral particle transport simulation suggests that installation of the additional baffle plates at the toroidal ends of the closed divertor components with vacuum pumping systems in the dome structure located near the baffle plates is quite effective for particle control and pumping in the LHD plasma periphery.

\section{Summary}

For studying an optimized closed divertor configuration, the effect of the additional baffle plates installed in both the toroidal ends of the closed divertor components near lower/upper ports is investigated in detail.

Magnetic field line tracing from the position on the LCFS shows that the additional baffle plates change the position of strike points from the lower/upper side to the inboard side of the torus by intersecting divertor legs similar to a target plate. The ratio of the number of strike points on the additional baffle plates to the total number of strike points in various magnetic configurations indicates that the additional baffle plates are most effective in the case where $R_{\text {ax }}$ is 3.60 m.

The neutral particle transport simulation with an onedimensional plasma fluid analysis of the divertor legs shows that the additional baffle plates locally raise the molecular hydrogen density. The molecular density is sufficiently high for efficient particle control. The investigation using the neutral particle transport simulation code proposes one possible candidate for an optimized closed divertor configuration, in which the additional baffle plates are installed in the toroidal ends of the closed divertor components with vacuum pumping systems located in the dome structure near the additional baffle plates. The fully three-dimensional neutral particle transport simulation is applied successfully for studying a closed divertor configuration for a three-dimensionally complicated magnetic field line structure in the LHD plasma periphery.

[1] N. Ohyabu et al., Phys. Rev. Lett. 97, 055002 (2006).

[2] M. Shoji et al., Contrib. Plasma Physics (to be published).

[3] A. Iwamae et al., Phys. Plasmas 12, 042501 (2005).

[4] S. Masuzaki et al., Nucl. Fusion 42, 750 (2002).

[5] M. Shoji et al., J. Nucl. Mater. 363, 827 (2007).

[6] Y. Feng et al., Plasma Phys. Control. Fusion 44, 611 (2002).

[7] P.C. Stangeby, The plasma boundary of magnetic fusion devices (Institute of Physics Publishing, Bristol and Philadelphia, 2000) p.423.

[8] T. Morisaki et al., J. Nucl. Mater. 313-316, 548 (2003).

[9] J. Spanier et al., Monte Carlo Principles and Neutron Transport Problem (Addison-Wesley Pub., Reading Mass, 1969) p.66.

[10] M. Kobayashi et al., J. Nucl. Mater. 350, 40 (2006).

[11] D. Reiter, Technical Report Jul-1947, KFA Juelich, Germany (1984). 